\title{
Caminhos cruzados: uma possível radicalização do YouTube Gamer
}

\author{
Crossing paths: a possible radicalization of YouTube \\ Gamer
}

\author{
Caminos cruzados: una posible radicalización \\ algorítmica de YouTube Gamer
}

\section{Francisco W. Kerche}

Mestrando em Sociologia e Antropologia do Programa de Pós-graduação em Sociologia e Antropologia (PPGSA-UFRJ).E-mail: chicowkn@gmail.com

\section{Miguel Borges Mendes}

Mestrando em Sociologia e Antropologia do Programa de Pós-graduação em Sociologia e Antropologia (PPGSA-UFRJ). E-mail migbmendes@gmail.com

\section{Resumo}

O seguinte trabalho procura analisar uma possível politização de vídeos sobre videogames partindo do algoritmo do YouTube, através de uma abordagem metodológica que combina a análise topográfica das redes de recomendação da própria plataforma e uma análise qualitativa de vídeos que poderiam ser responsáveis por ligar o tema a outros mais radicais. A proposta é testar a hipótese de que existe um processo de radicalização política encabeçado pelo algoritmo do YouTube. Ao contrário do que esperávamos, a plataforma se demonstrou muito capaz de dividir tematicamente suas recomendações, havendo pouca penetração de vídeos politizados em nossas extrações. Porém, fomos capazes de identificar estratégias de grupos mais politizados que foram utilizadas para se colocarem em espaços de recomendação.

Palavras-chave: YouTube; radicalização; algoritmos de recomendação; extrema direita. 


\section{Introdução}

Este trabalho é o resultado preliminar de um estudo experimental sobre radicalização política em redes sociais digitais. Nele, propomos aliar duas formas distintas de se analisar a comunicação política no YouTube: primeiramente, a partir de um olhar que chamaremos de topográfico, construímos redes de vídeos e canais por meio dos dados disponibilizados pela plataforma, observando as relações "à distância" (Moretti, 2008). Em segundo lugar, vamos analisar de forma qualitativa os vídeos que emergiram de nossa extração. Como forma de testar essa metodologia, nos debruçamos sobre a aliança entre produtores de conteúdo no YouTube sobre jogos de videogame e pessoas de posicionamento abertamente à direita do espectro político.

Esta escolha em particular partiu de uma rede construída por Kerche (2019), que encontrava um forte núcleo de canais de jogos próximo à rede da extrema-direita brasileira no YouTube. Escolhemos esta relação específica a fim de testar a hipótese de que a audiência destes canais que discutem temas aparentemente apolíticos, como jogos online, pode, pela interação com o algoritmo de recomendação da própria plataforma, aproximar-se de vídeos de orientação conservadora ou mesmo radical de direita. Para dar sentido a essa observação é necessário compreender o conceito de "algoritmo".

Algoritmos podem ser definidos como "processos codificados etapa-por-etapa implementados pelo YouTube para aumentar ou restringir a visibilidade.” (Bishop, 2019:2, tradução nossa). Em outras palavras, são um conjunto de códigos que criam critérios próprios para o bom funcionamento de uma plataforma. Dentre os diversos algoritmos que operam paralelamente no YouTube, encontramos algoritmos responsáveis pelas "tags" nos vídeos (Toderici et al., 2010), pela recomendação especializada para cada usuário (Baluja et al. 2008), para apresentar ou não vídeos na página inicial de cada usuário (Bishop 2019), dentre diversos outros. Por isso, ao discutirmos como $o$ algoritmo do YouTube pode gerar um processo de radicalização, referimo-nos apenas aos algoritmos de recomendação entre os vídeos, que apresentam uma lista de vídeos similares ao canto direito da tela do usuário.

O estudo busca partir de alguns youtubers gamers que produzem vídeos de streaming de jogos. Os vídeos costumam ser feitos com o jogo na tela principal e o apresentador conversando com o público, inclusive sobre assuntos que emergem pela interação dos usuários na aba de comentários. Mesmo esses vídeos sendo predominantemente de "gameplay" focados no funcionamento e acontecimentos do jogo, acabam sendo discutidos diversos temas envolvendo questões políticas. A hipótese que levantamos era de que as recomendações poderiam formar 
algum "pipeline" - um percurso de interação do usuário com a plataforma orientado pela interface - para vídeos mais politizados, com especial ênfase em canais radicalizados de extrema direita.

\section{Gamer-gate e a radicalização de usuários}

Existe hoje extensa bibliografia sobre como redes sociais podem acabar criando processos de radicalização em sua plataforma, muito devido aos seus algoritmos (Caiani e Kröll, 2015; O’Callaghan et al., 2015; Ribeiro et al., 2019). Também é um tema de grande impacto a radicalização política de direita partindo da ação de jovens gamers. Um exemplo bastante claro e bem documentado foi o movimento gamergate na radicalização política à direita nos Estados Unidos (Gerbaudo, 2018; Mortensen, 2018). O movimento tratou-se de uma revolta de gamers, organizados principalmente em sites como Reddit, Twitter e 4chan, contra a recepção majoritariamente positiva da crítica especializada a um jogo da desenvolvedora Zoe Quinn, que, alegavam, possuía relações pouco éticas com jornalistas. Foi, em resumo, uma campanha massiva de difamação a figuras femininas proeminentes na indústria de jogos, perpetrada majoritariamente por homens jovens insatisfeitos com uma suposta invasão do "progressismo" em um ambiente que assumiam que lhes fosse exclusivo (O’Donnell, 2020; Massanari, 2017; Braithwaite, 2016). Este era percebido de diversas formas, desde a maior presença e a dessexualização ${ }^{1}$ de personagens femininos até a inserção de temas caros à comunidade LGBTQIA+ em jogos.

Por mais que pareça uma situação bastante deslocada do contexto brasileiro, é importante notar como esse movimento foi fundamental para sedimentar as bases semânticas e ideológicas a partir das quais a autoproclamada alt-right se organizaria nas vésperas da eleição de Donald Trump. Por exemplo, o linguajar utilizado pelo ex-presidente para se referir pejorativamente àqueles engajados em lutas por justiça social, como snowflake ou safe spaces (flocos de neve e espaços seguros, respectivamente) foram gestados neste movimento (Bezio, 2018), assim como o termo Social Justice Warriors (guerreiros da justiça social). No Brasil, espaços semelhantes criaram o termo "mimimi", muito utilizado pelo presidente Bolsonaro e seus apoiadores de forma a minimizar movimentos e mesmo contestações de seus adversários políticos.

Além disso, é muito importante notarmos a proximidade demográfica dos dois movimentos, majoritariamente compostos por homens brancos, jovens, cisgênero e heterossexuais, que percebiam que estavam perdendo espaço político e econômico para minorias

\footnotetext{
${ }^{1}$ Era normal, por exemplo, a crítica a empresas que diminuíram o decote de personagens femininas de seus jogos.
} 
(O’Donnell, 2020). Talvez isso explique porque um dos difusores de mais visibilidade do gamergate tenha sido Milo Yiannopoulos, que escrevia para o portal Breitbart, à época gerenciado por Steve Bannon, estrategista da campanha de Trump (Bezio, 2018).

Nesse sentido, o movimento foi um caso exemplar de como um público organizado ao redor de um assunto superficialmente apolítico, como jogos de videogame (embora envolvesse diversos ressentimentos claramente atravessados politicamente), foi progressivamente instrumentalizado a partir da radicalização política. Inicialmente engajados em uma luta contra uma suposta falta de ética no jornalismo especializado, estes jovens progressivamente começaram uma campanha de perseguição e difamação a desenvolvedoras, jornalistas e pesquisadoras dedicadas à indústria. Vista como parte de uma luta justa, afirmava-se que era uma forma de defender uma masculinidade supostamente ameaçada pelo avanço do "progressismo" (O’Donnell, 2020). Por outro lado, esta ameaça era interpretada, em certos círculos já mais próximos da extrema direita, como parte de uma conspiração global que visava à destruição das bases morais do Ocidente (Mortensen, 2018).

\section{A politização do mundo}

Para compreender esse processo, partimos de dois marcos conceituais: a politização da vida e a radicalização política. Buscamos compreender se e, se sim, como conteúdos considerados "a-políticos" podem se conectar com outros mais abertamente à direita do espectro político. Para fazê-lo, partimos da similaridade do contexto contemporâneo brasileiro com experiências históricas, como o "gamergate" ou mesmo a ascensão do populismo de Margaret Thatcher (Hall, 1988). Propusemos uma forma de interpretar a disputa por espaço em plataformas de vídeo como o YouTube como um experimento de politização em um cenário de "guerra cultural” e de radicalização política.

Em seu ensaio "Todo Lado Tem Dois Lados"2, Rodrigo Nunes argumenta como a polarização política ${ }^{3}$, principalmente a partir da década de 1980 no Norte global, criou um terreno fértil para que a política passasse a ser disputada ao redor de temas morais. Para o autor, isso aconteceu, resumidamente, por conta de dois motivos. O primeiro diz respeito à própria

\footnotetext{
2 Disponível em https://www.revistaserrote.com.br/2020/06/todo-lado-tem-dois-lados-por-rodrigo-nunes/. Acesso em 26-06-2021.

${ }^{3}$ A existência de uma polarização política não é natural, nem deve ser vista sem questionamento sobre quais elementos são vistos como polarizados. Em consonância com Nunes e com estudiosos do tema, a enxergamos como sinal da efetividade dos discursos populista empregado pela direita, pelo menos desde o final dos anos 1980, em construir uma oposição entre uma esquerda que supostamente defende os privilégios de uma elite econômica e política a uma direita que valoriza a livre iniciativa individual (C.f. Benkler, 2018; Cesarino, 2019; Gerbaudo, 2014).
} 
natureza do tipo de cisão social que a polarização cria, onde dois polos são vistos de forma tão visceralmente inconciliáveis, que enxergam um ao outro como ameaças à sua própria existência. Por outro lado, alinhado a Fraser (2019), reconhece que o "fim da história" significou, por um breve momento, que o desenvolvimento econômico e político do neoliberalismo seria tratado como indiscutível e que, portanto, a disputa entre grupos políticos deveria ser travada em outras arenas $^{4}$. Nesse sentido, tudo se tornaria espaço para disputa política, menos a própria política.

Falando sobre a ascensão de Margaret Thatcher no Reino Unido, Stuart Hall (1988) argumenta como a comunicação da direita na imprensa britânica foi capaz de "preparar o terreno" para o populismo da dama de ferro ao instigar "pânicos morais" na opinião pública. Segundo o autor, a produção incessante de ameaças à sociedade britânica, principalmente a partir do "medo vermelho", foi um fator de unificação ao redor de uma pauta que aliava o conservadorismo moral com o liberalismo econômico. Para Hall (1988), o grande trunfo deste movimento estava em utilizar de temas aparentemente apolíticos para sedimentar o tipo de intervenção que um período de crise supostamente requereria. Em sentido semelhante, Cesarino (2020) destaca a efetividade da campanha do presidente Bolsonaro em colonizar politicamente espaços que não eram tratados desta forma como elemento de coesão, sendo a camisa amarela da seleção brasileira um dos principais exemplos dessa construção coletiva da coesão política sobre um mesmo tema.

Em um ambiente político e digital de constante disputa pela atenção dos usuários ${ }^{5}$, a radicalização política tende a resultar da busca por parcelas cada vez mais polarizadas do público (Lewis, 2018). Porém, é importante notar como essa radicalização é dada de forma assimétrica. Se Nunes (2020) ressalta como a polarização política tendeu a ser tencionada a partir de conservadores atacando temas que se supunham como pacificados (a busca por igualdade racial e de gênero, por exemplo), pesquisas sobre o tema mostram uma sobre-representação de veículos radicalizados à direita no ecossistema comunicacional contemporâneo, se comparados à esquerda (Benkler; Faris; Roberts, 2018).

Há uma ampla literatura que documenta a capacidade que as direitas têm de, através de estratégias específicas de comunicação em ambientes digitais, radicalizar sua base (Lewis, 2018; Davey; Ebner, 2017) - e, aqui, pouco importa se essas estratégias são empregadas de forma consciente ou não por todos os comunicadores. Elas se manifestam em diversos níveis, desde semânticos e ideológicos e até pelas estratégias de visibilidade próprias às plataformas

\footnotetext{
${ }^{4}$ É preciso ressaltar, porém, que este consenso não durou muito e que, talvez até pelo avanço de discursos que apostavam nesta polarização e na luta a partir de "guerras culturais", o neoliberalismo logo pôde voltar a ser posto em cheque, principalmente a partir da crítica ao chamado "globalismo".

${ }^{5}$ Atenção essa que significa mais engajamento, e, portanto, mais permanência na plataforma, resultando em mais lucros a partir de anúncios (Goldhaber, 1997).
} 
(aproveitar-se da visibilidade dada pelos algoritmos de recomendação das plataformas, utilizar determinada estética específica, mobilizar uma enorme variedade de tópicos para gerar engajamento, dentre diversos outros).

Do ponto de vista semântico, podemos pensar na sedimentação, através do uso cotidiano e da produção imagética de memes, de determinados enquadramentos para se tratar de certos temas. Por exemplo, houve um esforço documentado da direita nos Estados Unidos em transformar, discursivamente, os migrantes que iam para o país em uma "crise de refugiados", durante a corrida eleitoral de 2016 (Benkler; Faris; Roberts, 2018).

Quanto à experiência com a plataforma, uma das estratégias mais efetivas passa pela migração da audiência para canais mais radicalizados, principalmente a partir de indicações diretas ou pela participação destes produtores em canais voltados a grupos menos radicalizados ${ }^{6}$ (Lewis, 2018). No que diz respeito à ideologia, na pandemia experienciamos um exemplo claro de como as teorias da conspiração sobre sua origem e as formas de lidar com ela, mas também o receio relativo à vacinação, fomentaram o já crescente sentimento de desconfiança em relação a instituições governamentais (Machado; Siqueira; Gitahy, 2020).

\section{Mapas algorítmicos}

Partindo do que foi apresentado, buscamos observar como temáticas "neutras" ou mesmo não politizadas na internet podem ser levadas pelo algoritmo do YouTube para outras mais radicalizadas. Em suma, nossa hipótese inicial era de que canais de direita conseguiriam se apresentar em algum ponto em uma rede de recomendações de gamers famosos. Essa percepção parte de uma série de estudos sobre a capacidade da direita política, em especial de grupos mais radicalizados, de apresentar seu conteúdo com maior sucesso em redes sociais por meio da forma como são produzidos os algoritmos de recomendação.

É uma percepção que se tornou cada vez mais comum, introduzindo em diversos debates termos como "bolhas ideológicas", ou mesmo "tocas de coelho", que partem do princípio de que um usuário é apresentado a conteúdos cada vez mais extremistas, uma vez que a plataforma lida com sua interação positiva com esses conteúdos para introduzir cada vez mais conteúdos sobre a mesma perspectiva (O’Callaghan et al., 2015). O presente estudo não tem como foco observar o fenômeno de radicalização de usuários específicos por meio da repetição ou não de conteúdos radicalizados para eles, mas lidar com a introdução de conteúdos politizados e mesmo radicalizados em meio a temas não necessariamente políticos. Para isso, é necessário argumentar

\footnotetext{
${ }^{6}$ Para observar a efetividade de grupos de extrema-direita na radicalização de seus usuários ver Ribeiro (2019).
} 
o que pode ser considerado um conteúdo politizado, e como encontrá-lo em outros espaços no YouTube.

Em busca de observar se esse processo de radicalização partimos de um grupo inicial de jogos bastante populares no YouTube: League of Legends, Fallout e Counter Strike: Global Ofensive (CS:GO) e escolhemos um representante de grande impacto de cada. A proposta era montar um panorama dos youtubers mais relevantes para a plataforma no que tange o tema de games no Brasil. O método que utilizamos é chamado "bola de neve" (Goodman, 1961). Este parte de um grupo inicial de canais, para, a partir das relações que esses usuários podem possuir com outros, progressivamente produzir uma rede. No caso específico, expandimos os canais para outros que eram seguidos por eles na plataforma ${ }^{7}$. Em um segundo momento, foram extraídos todos os vídeos desse grupo inicial de canais e observadas todas as recomendações desses vídeos feitas pelo algoritmo do YouTube ${ }^{8}$. Entre eles, foi possível encontrar dois resultados bastante distintos.

Para ser possível apontar o que seriam os canais radicalizados ou mesmo de direita para nossa análise, era necessário construir o que seriam os grupos radicalizados localizáveis na amostra. Para fazer isso, foi repetido o procedimento metodológico apresentado por Kerche (2019). Utilizando o discurso de Jair Bolsonaro sobre "ótimas fontes de informação de direita no YouTube" durante a campanha presidencial de 2018, para construir um grupo de canais "sementes" 9 . Esses canais iniciais foram expandidos a partir de seus Featured Channels, ou seja, utilizando o YouTube Data Tools, encontramos todos os canais recomendados por esse grupo. Então, foram removidos todos os canais que não fossem de interesse e expandiu-se novamente a rede, pelo mesmo método, até que não se encontrasse mais nenhum canal relevante. Após a demarcação desse grupo final, foi feita uma última expansão partindo dos canais que esses youtubers seguiram. Com isso, a amostragem passou de 74 canais para 3.000. Esse grupo final foi recortado partindo da "centralidade do autovetor" (Alvarez-Socorro; Herrera-Almarza; González-Díaz, 2015). A medida permite observar o quão central é um canal levando em consideração a centralidade de seus vizinhos. Em outras palavras, não bastaria ter muitas

\footnotetext{
${ }^{7}$ Por exemplo, se o canal A segue os canais B, C e D, a extração vai encontrar as relações AB, AC e AD.

${ }^{8} \mathrm{O}$ download das recomendações e seguidores foi feito pelo site YouTube Data Tools (Rieder, 2015) e dos vídeos individualmente pelo pacote tuber, na linguagem R (Sood; Lyons, 2016). Todos os dados baixados no presente artigo foram feitos por esses dois programas, trabalhados em linguagem R com o pacote tidyverse (Wickham et al., 2019) e no software gephi (Bastian; Heymann; Jacomy, 2009).

${ }^{9}$ Canais sementes são canais que iniciam uma expansão em rede. Em outras palavras, é escolhida uma característica para localizar outros canais que se conectam com esse, como no caso o featured channels (canais que são recomendados por eles).
} 
conexões na rede, mas ter muitas conexões com outros youtubers que também têm muitas conexões ${ }^{10}$.

Primeiramente, entre os seguidores, já pudemos perceber percebemos uma grande quantidade de canais de direita. Dentre eles Jair Bolsonaro, Olavo de Carvalho, MamãeFalei, LiloVlog, entre outros. Porém, ao observar a rede de vídeos recomendados, foram encontrados pouquíssimos canais que não fossem diretamente relacionados com jogos. Dentre os com inclinação mais à direita, destacamos o programa de auditório "The Noite com Danilo Gentili”, como também o canal do Rafinha Bastos e o do pastor Silas Malafaia. A rede era fortemente dividida em três grandes agrupamentos - os quais chamamos de clusters - esses "clusters" são divididos por cores, e calculados por modularidade.

A modularidade é uma medida que indica que vídeos de uma mesma cor têm uma probabilidade maior de se conectar com outros vídeos dessa mesma cor do que se conectar com vídeos de qualquer outra cor. Esse cálculo permite compreender a construção da rede por grupos de similaridade. Algo central é como cada um dos grupos era estruturado em relação a um dos três jogos buscados inicialmente, o que demonstra uma tendência dos usuários de ver em sequência vídeos sobre algum jogo específico ${ }^{11}$. Essa primeira especialização de conteúdo, mesmo que outros temas conseguissem se apresentar, demonstra uma forte tendência do YouTube de segmentação de sua visibilidade e uma baixa introdução de temáticas distintas quando observado uma distância tão curta.

\footnotetext{
${ }^{10}$ A centralidade do autovetor permite pesar o quão central é um canal para a rede. Isso evita que canais muito grandes - chamados de gatekeepers - sejam considerados como muito relevantes para qualquer rede. Por exemplo, Felipe Neto, o maior YouTuber do Brasil e um dos maiores do mundo se encontra na rede, mas não é selecionado como um dos canais mais centrais, pois para os atores aqui presentes suas relações não são tão relevantes quanto outros como Jair Bolsonaro, canal mais central para toda a rede.

${ }^{11}$ A forte separação entre os clusters dividos para cada jogo demonstra de maneira direta o texto da própria empresa sobre a construção progressiva de tags entre os vídeos (Toderici et al., 2010). O texto demonstra como a plataforma utiliza algoritmos para analisar o conteúdo dos vídeos, tanto pelas imagens disponíveis quanto pelo som. Aqui, muito provavelmente dois principais fatores estão em jogo. Primeiro, uma análise de conteúdo, observando como jogos similares vão ser taxados de forma similar, a segunda, bastante fundamental para a plataforma, é que usuários interessados em um jogo provavelmente veem vídeos sobre o mesmo jogo em sequência, criando percursos de covisualização (Baluja et al., 2008).
} 
Figura 1: Expansão da rede inicial de canais

Cada expansão ocorria com os canais que falassem sobre jogos e fossem conectados aos canais de direita
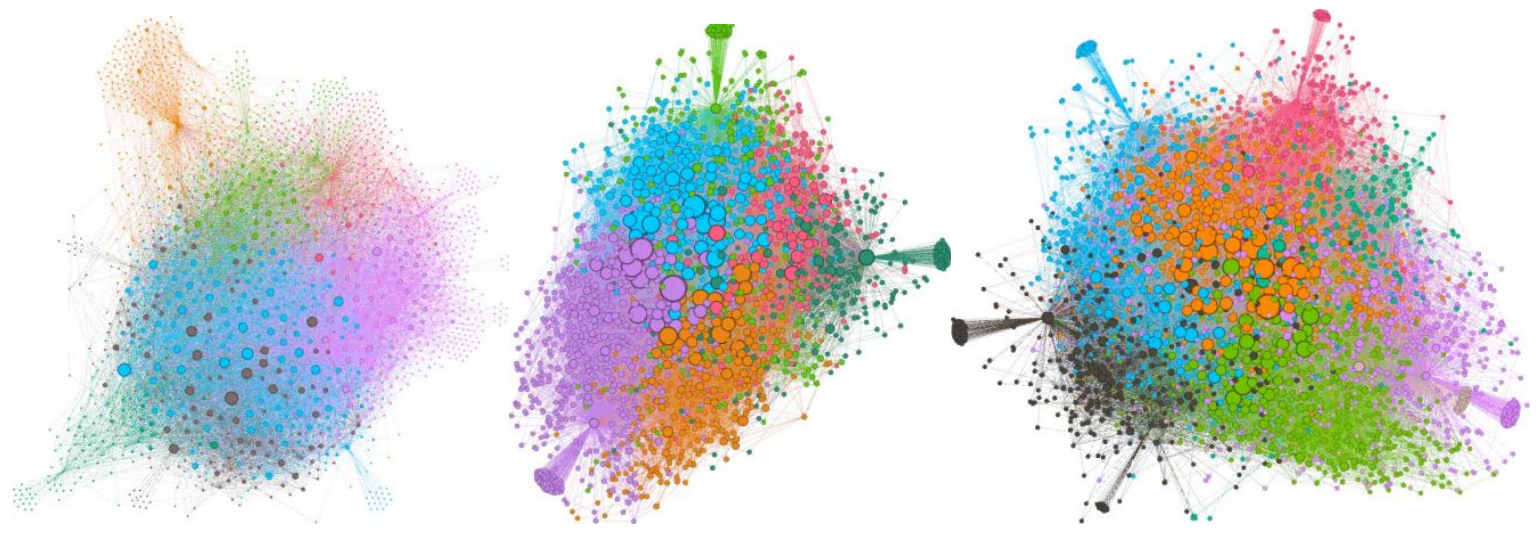

Uma primeira observação já demonstrava uma diferença entre os resultados dos seguidores de cada um desses grupos e das recomendações de vídeos construídas pelos algoritmos. Afinal, os grupos feitos pelos algoritmos eram muito mais endógenos do que aqueles feitos pelos seguidores. Decidimos então, construir um grupo inicial diferente e retornar às redes de seguidores, a fim de ampliar os canais iniciais. Para fazer parte dessa nova amostra era necessário cumprir três requisitos: (1) ser majoritariamente sobre video games, (2) ter ao menos 1000 seguidores e (3) seguir ao menos um canal radicalizado. Depois de selecionado o primeiro grupo, observamos seus seguidores, para encontrar mais canais que cumprissem os requisitos e assim repetidas vezes. Ao fim, foram encontrados 25 canais de gamers que cumpriam com os três critérios ${ }^{12}$. Partindo desse novo grupo, foi feito o mesmo procedimento metodológico. Os identificadores únicos (ID) de cada vídeo foram baixados e utilizados para observar as recomendações desses mesmos vídeos na plataforma. Partindo dessa rede, observamos quais canais de direita estavam presentes na amostragem. No caso, encontramos 17 canais de direita. Porém, o número é pouquíssimo expressivo, se considerarmos o total de 32.578 vídeos, dos quais apenas 40 seriam oriundos desse grupo, ou seja, $0.12 \%$ dos vídeos.

\section{Uma breve análise do jogo}

Antes de discutir a presença da direita, é importante fazer uma breve apresentação da atuação do algoritmo do YouTube. Baluja et al. (2008) apresenta um dos primeiros textos disponíveis pela Google sobre o funcionamento do algoritmo do YouTube. Originalmente, a

\footnotetext{
${ }^{12}$ A base utilizada para a última expansão se encontra em anexo ao fim do artigo.
} 
plataforma utilizava da co-visualização como uma medida para considerar relevante a recomendação entre videos ${ }^{13}$. Este método parte do princípio de que vídeos vistos juntos provavelmente discutem temas similares, e é utilizado hoje em diversos algoritmos, como o da Netflix, por exemplo (Halliman; Striphas, 2016). Porém, com o avanço das técnicas de aprendizado de máquinas como machine learning e deep learning as plataformas passaram a alterar a forma como selecionam seu conteúdo. Venturini explica que:

Ao invés de escrever uma equação complexa que iria unir usuários e vídeos, a computação é quebrada em dúzias de blocos de detecção, cada um considerando apenas uma característica do vídeo e do usuário. O output de cada um vira o input de um novo nível de computação e assim por diante por diversas camadas (às vezes com chamadas recursivas para níveis mais baixos) (Venturini, 2019:8).

Essa mudança nos modos de recomendação não impede que sejam separados por temas os vídeos, mas incentiva ainda mais essa fragmentação. Observações qualitativas como a de Bishop (2018) apresentam como os usuários têm ciência dessa fragmentação e constroem conteúdo altamente específico para sua audiência.

Vamos observar isso com mais profundidade na rede de recomendações de vídeos. Na figura 2, os nós (pontos) representam os vídeos, enquanto as arestas (linhas) representam as conexões formadas pelo algoritmo. As cores foram calculadas por modularidade, este cálculo parte de um algoritmo de construção de comunidades, que representa que a probabilidade de um vídeo se associar com outro em um mesmo grupo é maior do que se associar com algum vídeo aleatório na rede (Blondel et al., 2008). Um aspecto é como a rede encontrou como principal forma de divisão de comunidades os jogos que buscamos inicialmente. Nesse sentido, cada comunidade é focada majoritariamente em um jogo, e mesmo tendo alguns jogos que se distribuem em mais de uma, acabam encontrando sub-temáticas que são trabalhadas individualmente em cada uma. Os títulos de cada um dos grupos foi dado por nós, partindo de uma observação qualitativa dos vídeos mais relevantes presentes em cada agrupamento.

A relevância da rede é como ela é densamente populada por vídeos sobre video-games. Porém, não apenas. Mesmo que se construam interesses muito específicos, como um sub-grupo apenas sobre rap de league of legends, ainda existe espaço para vídeos sobre jogos nostálgicos e mesmo o eixo ao centro de "podcasts e programas". É perceptível como o usuário tem acesso a

\footnotetext{
${ }^{13}$ A explicação de Baluja é bastante técnica, e indica uma maneira para o algoritmo do YouTube compreender a distância entre vídeos sem recorrer a medidas de centralidade. Isso ocorre, pois medidas de centralidade são altamente custosas para o processamento computacional. A forma como isso ocorria era por meio de caminhos aleatórios por percursos de visualização dos usuários. Esses caminhos avançavam até encontrar uma "dummy value". Esse valor interrompia a programação e guardava a sequência de vídeos. Assim, um percurso de vídeos A-CH e outro A-T-S-W-F, outro D-W-X-A-R etc. poderiam construir a distância média de cada um dos vídeos presentes na plataforma com um baixíssimo custo. O algoritmo chamava-se adsorção (adsorption) e foi o início da introdução da co-visualização sem recorrer à análise de redes.
} 
novos tipos de conteúdo que possam interessá-lo e não apenas ao que já foi originalmente apresentado. Mesmo dentro do universo de referências de jogos, alguns novos jogos são eventualmente introduzidos na cadeia de recomendações, como "Red Dead Redemption 2".

Argumentamos que o algoritmo não necessariamente constrói um efeito bolha, o que é visível pela introdução de temas novos que podem ser de interesse do usuário, mesmo que isso não tenha relação com o grupo inicial. A construção do interesse feita algoritmicamente é pensada para além da repetição de um conteúdo bem recebido, mas pela introdução de uma gama nova de recomendações partindo da interação padronizada de diversos usuários. Mackenzie (2015) demonstra como essa "predição" de comportamentos é o verdadeiro interesse dos algoritmos de seleção de conteúdo.

Figura 2: Rede de recomendações de vídeos no YouTube,32.578 vídeos com 561.086 recomendações

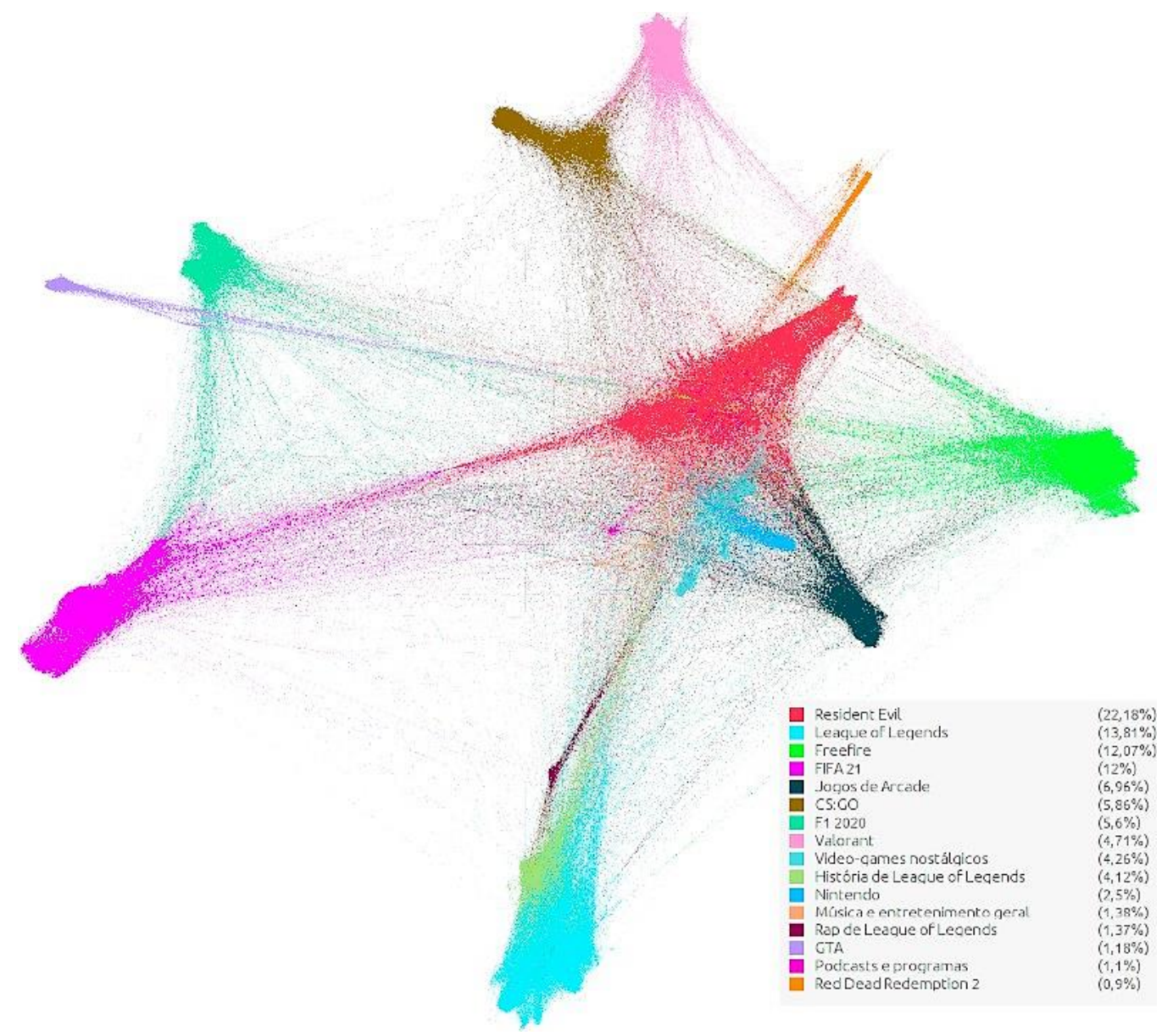

Um exemplo deste efeito, onde temas distintos dos originalmente buscados emergem nas redes de recomendação é justamente o fato de que vídeos mais politizados aparecem em uma amostra construída partindo de canais voltados a jogos. Na figura 3, os nós em amarelo indicam 
onde estão localizados os vídeos de canais alinhados com a direita brasileira, identificados, como já foi explicado previamente, a partir de um data-set de canais de direita construído partindo dos canais recomendados por Jair Bolsonaro como "ótimas fontes de informação no YouTube". Torna-se perceptível como esses canais estão majoritariamente distribuídos no eixo de "programas e podcasts". Também é bastante relevante como alguns dos vídeos aparecem no grupo de games nostálgicos, que demonstra a introdução da nostalgia como uma temática que pode ser apropriada pelo grupo para discutir temas mais conservadores.

Mesmo que a quantidade de vídeos que podem ser considerados de direita seja quase marginal para a rede, propomos observar com mais profundidade quais vídeos conseguiram ser associados à ela, para assim entender até que ponto e de que forma alguns vídeos políticos conseguiram expandir para novos horizontes algorítmicos.

Figura 3: Rede de recomendações de vídeos com destaque os vídeos de direita (em amarelo)

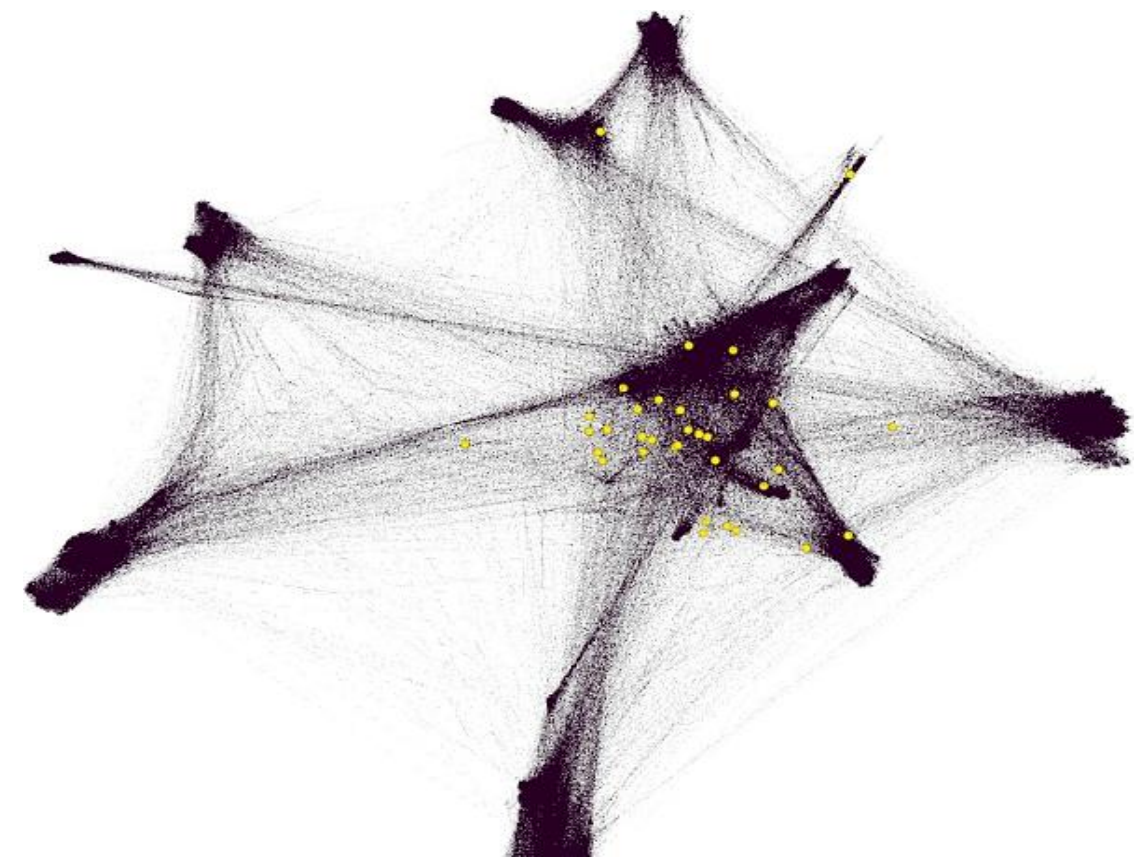

\section{Pontes de radicalização e os pesquisadores como objeto}

O estudo do algoritmo do YouTube passa obrigatoriamente pela dificuldade de distinguir quais de seus efeitos podem ser atribuídos a ele. Não é possível responsabilizar sua operação apenas aos aspectos técnicos e à ação de cientistas de dados que o constrói. Argumentamos que entender a atuação de algoritmos parte também por compreender como os usuários interagem com eles. Como afirma Sophie Bishop (2019), os usuários são ao menos parcialmente cientes 
dos algoritmos de recomendação que organizam sua navegação. Um bom exemplo apresentado pela autora é de como youtubers de maquiagem faziam "fofocas algorítmicas" para discutir formas de viralização dentro da plataforma do YouTube. O distanciamento dessas youtubers do aspecto técnico da operação dos algoritmos não as impediam de produzir um conhecimento altamente experimental e mesmo lidar com as possibilidades e limites do algoritmo de maneira empírica. Afinal, é na interação dos usuários com a plataforma, e seus algoritmos que é possível verdadeiramente compreender como são feitas essas seleções.

Ao utilizar os padrões de interação dos usuários, o algoritmo encontra percursos por vezes não óbvios para montar recomendações. Cabe aos analistas dar sentido ao que é feito por esses algoritmos (Kotliar, 2020). Para discutir o papel dos atores políticos envolvidos na rede de jogos, é importante também discutir o papel dos pesquisadores. A escolha de extração de dados foi feita a fim de analisar se ocorre um efeito de radicalização no YouTube de games. Isso parte tanto da observação da atuação política de coletivos de games pelo mundo, quanto pela análise do processo de radicalização na própria plataforma. Porém, para construir o grupo base, partimos sempre de usuários que tivessem alguma escolha que os aproximasse de grupos extremistas.

Como apresentamos anteriormente, apenas selecionamos youtubers que seguissem ao menos um canal considerado radicalizado. Um efeito que deve ser considerado é como a escolha dos youtubers sobre quem seguir também influencia na atuação dos algoritmos. Essa pergunta ainda não será respondida, mas introduz camadas de possibilidade para pensar o papel humano na construção das recomendações do YouTube. Logo, a extração foi feita em busca de canais que pudessem criar essas pontes. A presença dos vídeos de direita não pode ser vista como prova cabal da radicalização de usuários por influência exclusiva plataforma. Porém, propomos usá-la como ponto de partida para tentar compreender quais tipos de conteúdos mobilizados por esses grupos têm a capacidade de ser colocados em ambientes que não são necessariamente óbvios.

A segunda parte do estudo busca justamente compreender o porquê da introdução dos vídeos de direita na amostragem, e especialmente qual o aspecto estratégico ou experimental que fez com que esses grupos conseguissem ser introduzidos nessa rede.

\section{Construindo pontes}

Um dos atores centrais entre os youtubers encontrados é Nando Moura, youtuber que hoje encontra-se em forte desacordo com Jair Bolsonaro, mas que foi muito relevante para a eleição do presidente em 2018. Nando Moura aparece em 3 vídeos na amostragem, um deles 
chama-se "Vamos jogar um PREIXTEIXO????"14, em que o youtuber, em um dia de chuva, apresenta quais jogos tem em seu videogame e comenta cada um deles. Outro vídeo do mesmo criador de conteúdo é "Kong x Godzilla - Ei Nerd está ERRADO!",15, em que Moura discute suas expectativas para o filme Kong x Godzilla argumentando sobre como o maior canal de cultura nerd do Brasil, "Ei Nerd”, estaria errado de estar animado com o lançamento do filme.

Um primeiro fator é que o forte apelo chamativo bem claro de ambos os vídeos, com uma linguagem bastante informal, como o termo "PREIXTEIXO", para se referir ao console playstation, ou mesmo a direta relação com o canal Ei Nerd não parecem estar no título por acaso. Moura discute de maneira muito direta temas da cultura pop com uma linguagem informal e acessível e, mesmo tendo a maior parte de seu foco em questões políticas, faz propaganda sobre curso de línguas, discute videogames e cultura nerd como um todo. Algo que se mostra claro é como seus vídeos com teor mais político não conseguiram entrar nas recomendações que extraímos partindo de canais de jogos, porém, os que focam em temas da cultura nerd, ou abertamente sobre vídeo games, encontraram uma entrada possível na amostragem. A estratégia permite que usuários que não estão acostumados com o discurso do youtuber, passem a consumir seu conteúdo sobre jogos e depois, caso se acostumem e se interessem pelo seu perfil, passem a ver vídeos de todos os temas.

É curioso notar como, mesmo em um vídeo em que comenta os jogos de videogame de sua coleção, Moura se utiliza de um linguajar próprio da direita na internet para falar sobre eles. Por exemplo, reclama de um excesso de "lacração" em um dos jogos, ou como a protagonista de outro é uma "feminista louca". Há aqui ecos claros de demandas dos jovens irritados durante o gamer-gate. Por outro lado, antes de comentar o trailer de Kong x Godzilla, reclama como as pessoas gastam sua atenção em filmes de baixa qualidade, ao invés de discutir os problemas do país, casualmente acenando para outra editoria de seu canal.

Outro vídeo que fala de forma geral sobre vídeo games que emergiu em nossa amostra é o "PLAYSTATION 5 vs. XBOX SERIES X - Quem é o Melhor?"16, do canal "Você Sabia?”. O vídeo é uma comparação geral sobre os dois consoles, mas chama a atenção a forma como os apresentadores reclamam do preço que os dois provavelmente custarão no Brasil. Se por um lado reclamam de forma um tanto apolítica, sem apontar culpados, da alta do dólar, são bastante incisivos ao reclamar dos impostos que incidem sobre a importação de eletrônicos no país. Um

\footnotetext{
${ }^{14}$ Disponível em https://www.youtube.com/watch?v=fDoacekvh5s. Acesso em 26-06-2021.

${ }^{15}$ Disponível em https://www.youtube.com/watch?v=I1CiX5H5mTs. Acesso em 26-06-2021.

${ }^{16} \mathrm{O}$ caminho que liga a insatisfação tributária em relação ao preço de jogos eletrônicos no país e um forte movimento libertário, no sentido austríaco da palavra, na internet brasileira merece ser desenvolvido no futuro.
} 
dos comentários do vídeo sintetiza este sentimento: "Olhar o preço das coisas no Brasil só me prova uma coisa, "IMPOSTO É ROUBO! ${ }^{17 "}$.

O canal "Hoje no Mundo Militar" é outro já bem próximo ao universo temático da direita que aparece na rede. Como o nome indica, é um canal dedicado a "temas militares atuais". Um dos três vídeos do canal que apareceram em nossa amostra se chama "Se Taiwan for atacada pela China, agiremos juntos para defender aquela democracia!', EUA e Japão"18 e fala sobre uma resposta estadunidense e japonesa a uma possível invasão chinesa a Taiwan. O vídeo parte de pressupostos que estão em consonâncias com o ideário da direita sobre a política internacional chinesa: que os EUA seriam os responsáveis por resguardar Taiwan, que a China não teria nenhuma ingerência sobre a ilha ${ }^{19}$ e que o país estaria se preparando para uma política expansionista no leste asiático. Se os sentimentos anti-chineses aparecem apenas de forma implícita, os comentaristas do canal são menos sutis. Um deles, por exemplo, culpa o país pelo surgimento do coronavírus: "Essa China está se tornando um problema mundial, primeiro contaminam o mundo com o vírus nojento deles agora querem tomar terras alheias! Todos sabemos como isso termina!".

Nossa rede conta também com canais explicitamente de direita, como o "Te Atualizei!" e o canal do Mauro Fagundes. Ambos fazem parte do que poderia se chamar de rede governista de produtores de conteúdo, são muito próximos do presidente Bolsonaro e de seus filhos e são (ex)alunos de Olavo de Carvalho. Na rede aparecem um vídeo de cada um destes canais, ambos tecendo críticas ao Superior Tribunal Federal (STF). Além deles, aparecem também o canal "Mamãe Falei" e o do Gabriel Monteiro, ligados ao Movimento Brasil Livre (MBL). Outra figura próxima ao presidente Bolsonaro que aparece na rede é o padre conservador Paulo Ricardo, embora o vídeo em específico seja uma homilia diária do padre. Ainda no tema religioso, aparece também na rede um episódio do especial de natal da produtora "Brasil Paralelo", uma grande difusora de revisionismo histórico à direita. Há ainda um vídeo do canal "Foco do Brasil", que se coloca com um canal de notícias da mídia tradicional, mas é abertamente favorável ao governo ${ }^{20}$.

Em relação à quantidade de vídeos, os conteúdos da rede de jornalismo Jovem Pan, consideravelmente próxima ao governo Bolsonaro, são os mais presentes. No total, distribuídos

\footnotetext{
${ }^{17}$ Disponível em https://www.youtube.com/watch?v=dLIr1UQcxNQ. Acessado em 26-06-2021.

${ }^{18}$ Disponível em https://www.youtube.com/watch?v=CTd0OmrUG34. Acessado em 26-06-2021.

${ }^{19}$ A independência de Taiwan e de Hong Kong frente à China vem ganhando muita importância para a direita brasileira, ainda mais durante a pandemia de coronavírus.

${ }^{20}$ É uma estratégia amplamente documentada de radicalização de conteúdo canais na internet disfarçarem propaganda política de apresentação fria de notícias, emulando esteticamente programas jornalísticos de televisão (Lewis, 2018).
} 
entre o canal principal da emissora e os específicos dos programas "Pingos nos Is", do "Pânico na Jovem Pan" e do "Morning Show", são treze vídeos. Destes, três são transmissões de jogos de futebol e uma entrevista exclusiva concedida pelo presidente Bolsonaro nos últimos dias de janeiro de 2021. Nela, o presidente discorre sobre os feitos de seu governo durante a pandemia, põe em dúvida a eficácia de vacinas, exalta a da cloroquina e volta a afirmar que ganhou as eleições de 2018 no primeiro turno. Também agradece mais de uma vez o espaço que recebe na "mídia independente", ao contrário dos supostos ataques que recebe constantemente da "mídia tradicional”.

\section{Uma nova forma de disputa}

A presença dos vídeos de direita entre a rede dá algumas pistas sobre aspectos da radicalização na internet. Porém, a amostragem de 32.000 canais é relativamente pequena para poder generalizar qualquer aprendizado estatístico, mesmo que nos dê bons indicativos do funcionamento da disputa política digital.

Enquanto não temos dados significativos para afirmar até que ponto a escolha dos usuários de seguir os canais de direita foi o que impulsionou sua presença na amostragem, podemos observar alguns aspectos presentes nos vídeos para a introdução desses no grupo. A disputa digital se dá por uma mesma regra para qualquer conteúdo. De maneira geral, o algoritmo opera "da mesma forma" para organizar conteúdos sobre jogos e política: por meio das interações dos usuários com a plataforma. Esse formato incentiva que usuários tenham mais engajamento, inclusive por meio da monetização - vendendo espaços de propaganda nos vídeos para empresas. A particularidade do algoritmo é conseguir criar blocos de detecção que permitam selecionar, para um perfil específico, o vídeo com o maior potencial de ser clicado. Isso parte tanto do conteúdo específico do vídeo, quanto com os padrões de interação que outros usuários já possam ter tido com o vídeo em questão ${ }^{21}$.

Bishop (2018) apresenta algumas estratégias utilizadas por youtubers para serem bem recomendados pelo algoritmo, como mimetizar conteúdo de youtubers mais famosos, ser colocado em playlists já consolidadas em um percurso que passe por youtubers relevantes, dentre diversas outras. O que encontramos aqui são usos dessas estratégias pelos influencers, especialmente Nando Moura. O vídeo de Moura sobre Godzilla o permite discutir diretamente

\footnotetext{
${ }^{21}$ Um bom exemplo é que se um usuário está vendo um vídeo sobre CS:GO, existe uma probabilidade grande que tenha interesse no jogo. $\mathrm{O}$ algoritmo aproveita a informação de milhões de usuários que se interessam pelo mesmo tema para padronizar o que será visto por esse usuário. É um uso probabilístico desses valores para montar um perfil de usuário e um percurso de utilização.
} 
com o canal mais influente no dia, possivelmente sendo colocado em listas de reprodução junto com o canal.

Outro método, utilizado por Nando Moura e outros, como MamãeFalei, é o chamado clickbait (Venturini, 2019). O clickbait é uma forma de construção de títulos bastante chamativos sobre temas que não sejam necessariamente os mesmos dos vídeos. Essa é uma forma que se popularizou pela maneira de monetização da plataforma. Isso parte de uma premissa lógica, cada clique permite a venda dos dados sobre quem clicou, logo uma estratégia que vise maximizar os cliques, também maximizaria o retorno. Um bom exemplo de clickbait pode ser visto no canal MamãeFalei com o vídeo “O Discurso Que Me Arrepiou”. Nele, Arthur do Val, apresentador do canal e deputado federal de São Paulo, apresenta o discurso de Kim Kataguiri contra Jair Bolsonaro dentro da Câmara, o aspecto chamativo e pouco esclarecedor do título é pensado de forma a atrair o usuário.

O que é levado em conta para um vídeo ser ou não bem recomendado pela plataforma são modelos de engajamento que ela consiga mobilizar. Dentre esses estão: títulos chamativos, boas tags, estar em boas sequências de recomendação, discutir temas bastante procurados, ter muitos comentários e likes, dentre diversos outros. Isso cria em certa medida uma homogeneização do conteúdo, tanto pela busca pela mímese do formato de vídeo de usuários já bem estabelecidos, quanto pela tentativa de discutir temas de grande visibilidade para ganhar mais visibilidade (Bishop, 2018). Argumentamos que a pluralidade dos temas abordados pela direita política, apoiados com o formato de "clickbait" e referências diretas a vídeos e canais mais conhecidos, permite criar pontes de recomendação com conteúdos não óbvios.

O uso de estratégias voltadas ao algoritmo acaba encontrando uma percepção dupla sobre a plataforma e sobre a disputa política. Enquanto ela é um cenário de forte relevância para a chamada "campanha não oficial", que se define pela

[...] criação e disseminação de mensagens por atores que se situam fora da estrutura partidária das alianças eleitorais e derivam seu capital comunicacional do potencial de gerar conteúdo, criar conexões em rede e atrair atenção nas plataformas digitais (Santos Junior 2019:43).

Também é fonte de renda para aqueles que produzem conteúdo em seu interior ${ }^{22}$. Essas duas esferas da produção de conteúdo não são opostas, mas complementares. Afinal, a ampliação do público ouvinte, a busca por bons espaços de recomendação e mesmo a tentativa de introdução em novos espaços, como aqui listado o universo gamer, são paralelamente formas de

\footnotetext{
22 A desmonetização e possivelmente a deplataformização são prática de limitação para discurso de ódio e fake news na plataforma. Essas formas de limitar conteúdo extremista tem ganhado cada vez mais atenção, e acaba expondo a importância da monetização para a produção de conteúdo político dos chamados influencers (Rogers, 2020). Um exemplo disso é a desmonetização ocorreu com o youtuber Nando Moura em Fevereiro de 2019.
} 
melhorar o canal como um "negócio", como também de ampliar a visibilidade da disputa cultural mobilizada por esses atores.

\section{Considerações finais}

Ao nos propormos a esta pesquisa, pensávamos em duas possibilidades: ou observaríamos um forte pipeline para a radicalização, ou estaríamos tão determinados a encontrá-lo que acabaríamos produzindo resultados ad hoc nesse sentido. A discussão pública e acadêmica sobre o assunto e a nossa experiência pareciam indicar um caminho claro: processos algorítmicos de recomendação de conteúdo preparam ideologicamente uma geração de homens jovens brasileiros para radicalização política à direita, principalmente partindo de círculos ligados a jogos eletrônicos, levando-os como que cada vez mais para dentro da "toca do coelho" da extrema-direita (Lewis, 2018; O’Callaghan et al., 2015).

Porém, quando elaboramos formas de testar esta hipótese, descobrimos que esse caminho, por mais que aparentemente exista, é bem menos transparente do que poderíamos supor. Se imaginávamos que a ocupação de espaços digitais pela direita era tão efetiva a ponto de tornar-se incontornável nos caminhos de recomendação algorítmica da plataforma, descobrimos que o YouTube é surpreendentemente eficaz em separar tematicamente o conteúdo que apresenta a seus usuários, mesmo quando estes temas aparentemente são produzidos visando públicos semelhantes. Descobrimos também um limite de uma pesquisa realizada assim, “à distância", com ferramentas de extração de dados que interagem com as plataformas sem levar em conta o viés dos usuários.

Os temas, a presença dos termos de direita e mesmo as estratégias são frutos de uma forma comunicativa entre usuário e algoritmo. Outros estudos devem ser pensados buscando lidar com a particularidade da orientação dos resultados com vistas aos usuários. Todavia, a observação feita a partir da plataforma YouTube Data Tools, que extrai os dados diretamente da API, como se fosse um usuário que ainda não teve contato com nenhum conteúdo na plataforma, permitiu analisar de maneira mais explícita como são feitas as disputas políticas em redes sociais, em especial no YouTube ${ }^{23}$.

Nossa análise pôde observar as "pontes" produzidas por canais radicalizados para se introduzirem em uma temática "neutra", e com isso fomos capazes de identificar algumas das estratégias que lhes permitiram ganhar espaço nesta parte da plataforma. Quanto às formas de

\footnotetext{
${ }^{23}$ É importante ressaltar que mesmo a extração da API ainda lida com algumas determinações como o endereço de IP do extrator. Indicando, por exemplo, que pode haver alguma alteração dos resultados baseado no local de extração inicial. A tentativa de construir como uma base "neutra" é sempre limitada e quase impossível dentro das redes sociais.
} 
disputa cultural, mesmo os vídeos aparentemente mais imparciais encontram forte referência ao universo semântico à direita, como no caso do vídeo do canal "Você Sabia?" onde os apresentadores acenavam ao slogan "imposto é roubo", ou quando Nando Moura reclamou do excesso de "lacração" em um jogo protagonizado por uma mulher. Nesse sentido, talvez o maior êxito destes produtores esteja em sedimentar representações políticas a partir de temas aparentemente apolíticos, como o preço de determinado console. Desta forma, usuários cativados por este discurso poderiam, eventualmente, procurar outros conteúdos alinhados a ele. Uma possível interpretação sobre isso é que o caminho que leva à radicalização política, geralmente atribuído exclusivamente às recomendações da própria plataforma, pode ser resultado de afinidades construídas pelos e nos próprios usuários.

Fundamentalmente, a própria separação entre o efeito do algoritmo e dos usuários se torna progressivamente mais frágil. A interação dos usuários com o conteúdo digital é fundamental para o funcionamento da plataforma, uma separação estanque entre produtores de conteúdo e consumidores se torna cada vez mais obsoleta. Observar as formas pelas quais os canais de direita se apresentam em recomendações algorítmicas sobre jogos, criando conteúdos acessíveis ao público ouvinte como um todo, permite entender a tentativa de diálogo performático entre usuário e algoritmo. Como argumentamos, os usuários criam relações comunicativas com seus algoritmos, tanto para serem melhores recomendados, quanto para terem melhores recomendações. Argumentamos como uma crítica que incida apenas sobre o funcionamento do algoritmo pode deixar de lado os aspectos estratégicos, como também experimentais, que os atores podem vir a mobilizar para conseguir ostentar boas recomendações e serem introduzidos para novos grupos dentro da plataforma. A percepção da radicalização política na plataforma do YouTube deve ser observada tanto pela ação dos algoritmos per se, quanto dos próprios usuários em relação a esses algoritmos.

\section{Referências}

ALVAREZ-SOCORRO, Alejandro; HERRERA-ALMARZA, Gioconda; GONZÁLEZ-DÍAZ, L. (2015), "Eigencentrality based on dissimilarity measures reveals central nodes in complex networks". Scientific reports 5:17095.

BALUJA, Shumeet et al (2008), "Video suggestion and discovery for youtube: taking random walks through the view graph". Proceedings of the 17th international conference on World Wide Web, pp. 895-904.

BASTIAN, Mathieu; HEYMANN, Sebastien; JACOMY, Mathieu (2009), "Gephi: an open source software for exploring and manipulating networks", in Proceedings of the International AAAI Conference on Web and Social Media, [S.L] v. 3. Issue 1. 
BENKLER, Yochai; FARIS, Robert; ROBERTS, Hal (2018), Network propaganda: Manipulation, disinformation, and radicalization in American politics. Nova Yorl, NY, Oxford University Press.

BEZIO, Kristin MS (2018), "Ctrl-Alt-Del: GamerGate as a precursor to the rise of the alt-right". Leadership, v. 14, n. 5, pp. 556-566 [Consult. 12-11-2021]. Disponível em https://doi.org/10.1177/1742715018793744

BISHOP, Sophie (2019), "Managing visibility on YouTube through algorithmic gossip". New Media \& Society, v. 21, n. 11-12, pp. 2589-2606 [Consult. 12-11-2021]. Disponível em https://doi.org/10.1177/1461444819854731

BISHOP, Sophie (2018), "Anxiety, Panic and Self-Optimization: Inequalities and the YouTube Algorithm". Convergence: The International Journal of Research into New Media Technologies v. 24, n. 1, pp. 69-84 [Consult. 12-11-2021]. Disponível em https://doi.org/10.1177/1354856517736978

BLONDEL, Vincent D.; GUILLAUME, Jean-Loup; LAMBIOTTE, Renaud; LEFEBVRE, Etienne (2008), "Fast unfolding of communities in large networks". Journal of statistical mechanics: theory and experiment, v. 10.

BRAITHWAITE, Andrea (2016), "It's About Ethics in Games Journalism? Gamergaters and Geek Masculinity". Social Media + Society, v. 2, n. 4 [Consult. 12-11-2021]. Disponível em https://doi.org/10.1177/2056305116672484

CAIANI, Manuela; KRÖLL, Patricia (2015), "The transnationalization of the extreme right and the use of the Internet". International Journal of Comparative and Applied Criminal Justice v. 39, n. 4, pp. 331-51[Consult. 12-11-2021]. Disponível em https://doi.org/10.1080/01924036.2014.973050

CESARINO, Letícia (2020), "Como vencer uma eleição sem sair de casa”. internet\&sociedade, v. 1, n. 1, pp. 91-120.

CESARINO, Letícia (2019), "Identidade e representação no bolsonarismo: Corpo digital do rei, bivalência conservadorismo-neoliberalismo e pessoa fractal". Revista de Antropologia, v. 62, n. 3, pp. 530-557 [Consult. 12-10-2021]. Disponível em https://www.revistas.usp.br/ra/article/view/165232

DAVEY, Jacob; EBNER, Julia (2017), The Fringe Insurgency: Connectivity, Convergence and Mainstreaming of the Extreme Right. London.

ESPOSITO, Elena (2017), "Artificial communication? The production of contingency by algorithms". Zeitschrift für Soziologie v. 46, n. 4, pp. 249-65.

FRASER, Nancy; SUNKARA, Bhaskar (2019), The old is dying and the new cannot be born: from progressive neoliberalism to Trump and beyond. London; New York, Verso. OCLC: on1037290135.

GERBAUDO, Paolo (2014), "Populism 2.0: Social media activism, the generic Internet user and interactive direct democracy". Social media, politics and the state. Routledge, pp. 79-99.

GERBAUDO, Paolo (2018), "Social media and populism: an elective affinity?". Media, Culture \& Society, v. 40, n. 5, pp. 745-753 [Consult. 12-11-2021]. Disponível em https://doi.org/10.1177/0163443718772192

GOLDHABER, Michael H. (1997) The attention economy and the Net. First Monday, v. 2, n. 4 [Consult. 01-10-2021]. Disponível em http://journals.uic.edu/ojs/index.php/fm/article/view/519

GOODMAN, Leo A (1961), "Snowball sampling”. The annals of mathematical statistics, pp.148-70. 
HALL, Stuart (1988), The hard road to renewal: Thatcherism and the crisis of the left. London; New York, Verso.

HALLIMAN, Blake; STRIPHAS, Ted (2016), "Recommended for you: The Netflix Prize and the production of algorithmic culture". New Media \& Society v. 18, n. 1, pp. 117-37.

KERCHE, Francisco W. (2019), “As Redes do Conservadorismo Brasileiro: Mapeando a Nova Direita no youtube”, In VI Simpósio Internacional do LAVITS 17.

KOTLIAR, Dan M. (2020), "The return of the social: Algorithmic identity in an age of symbolic demise". New Media \& Society, v. 22, n. 7, pp. 1152-67.

LEWIS, Rebecca (2018), “Alternative influence: Broadcasting the reactionary right on YouTube". Data \& Society 18.

MACHADO, Dayane Fumiyo Tokojima; SIQUEIRA, Alexandre Fioravante de; GITAHY, Leda (2020), "Natural Stings: Selling Distrust About Vaccines on Brazilian YouTube". Frontiers in Communication, v. 5 [Consult. 05-11-2021]. Disponível em https://www.frontiersin.org/articles/10.3389/fcomm.2020.577941/full

MASSANARI, Adrienne (2017), “\#Gamergate and The Fappening: How Reddit's algorithm, governance, and culture support toxic technocultures". New Media \& Society, v. 19, n. 3, pp. 329-346 [Consult. 1211-2021]. Disponível em http://journals.sagepub.com/doi/10.1177/1461444815608807

MORTENSEN, Torill Elvira (2018), "Anger, Fear, and Games: The Long Event of \#GamerGate". Games and Culture, v. 13, n. 8, pp. 787-806. [Consult. 12-11-2021]. Disponível em https://doi.org/10.1177/1555412016640408

MORETTI, Franco (2008), A literatura vista de longe. Porto Alegre, PA, Arquipélago Editorial.

O'CALLAGHAN, Derek et al. (2015), "Down the (white) rabbit hole: The extreme right and online recommender systems". Social Science Computer Review, v. 33, n. 4, pp. 459-478. Sage Publications Sage CA, Los Angeles, CA.

O'CALLAGHAN, Derek et al. (2015), "Down the (white) rabbit hole: The extreme right and online recommender systems". Social Science Computer Review, v. 33, n. 4, pp. 459-78.

O'DONNELL, Jessica (2020) "Militant meninism: the militaristic discourse of Gamergate and Men's Rights Activism". Media, Culture \& Society, v. 42, n. 5, pp. 654-674. [Consult. 12-11-2021]. Disponível em https://doi.org/10.1177/0163443719876624

RIBEIRO, Manoel H. et al (2019), "Auditing Radicalization Pathways on YouTube". arXiv:1908.08313 [cs], dez. 2019. ArXiv: 1908.08313 [Consult. 12-11-2021]. Disponível em http://arxiv.org/abs/1908.08313

RIEDER, Bernhard (2015), YouTube Data Tools (Version 1.10) [Software]. Retrieved from YouTube Data Tools website: https://tools. digitalmethods. net/netvizz/youtube.

ROGERS, Richard (2020), "Deplatforming: Following extreme Internet celebrities to Telegram and alternative social media". European Journal of Communication, v. 35, n. 3, pp. 213-229 [Consult. 1211-2021]. Disponível em https://doi.org/10.1177/0267323120922066

SANTOS JUNIOR, Marcelo Alves dos (2019), "\#VaipraCuba! a gênese das redes de direita no facebook". Curitiba, Appris Editora.

SOOD, Gaurav; LYONS, Kate (2016), Package 'tuber'. [S.1.]. 
TODERICI, George et al. (2010), "Finding meaning on youtube: Tag recommendation and category discovery". pp. 3447-54 IEEE

VENTURINI, Tommaso (2019), "From Fake to Junk News, the Data Politics of Online Virality", in Bigo, D.; Isin, E.; Ruppert, E. (Eds.). Data Politics: Worlds, Subjects, Rights. Routledge [Consult. 12-112021]. Disponível em https://hal.archives-ouvertes.fr/hal-02003893

WICKHAM, Hadley (2019) et al. "Welcome to the tidyverse". Journal of Open Source Software, v. 4, n. 43, pp. 1686. 


\section{Anexo metodológico}

Tabela 1: Base de canais encontrados para última extração, ordenado pela quantidade de seguidores

\begin{tabular}{|c|c|c|}
\hline Id & Canal & inscritos \\
\hline UCPmKDdmH15F7EvJGFoSHOwg & Fiaspo & 3980000 \\
\hline UCsTpmc6NjJzUSUiOA1WA0OA & DMenor11 & 2170000 \\
\hline UCHRXZPV3C2JfgS4udB9Wh7w & Um Gamer Aleatório & 1440000 \\
\hline UCxS15tW33vInzsJFCnlTOMQ & VHTheGamer BR & 1250000 \\
\hline UCexaxaj4QzEirjgmPCBdhSg & Jiraiya & 875000 \\
\hline UC-bt2BScQptIFvRa_m8RyTg & Robertinha & 750000 \\
\hline UCdgiFX-1fIlO8BScaDD8Xw & MuriloRT & 476000 \\
\hline UCGzgzPIDIRkoXjRmjNBmFQA & THG NOÉ & 423000 \\
\hline UCkG9YvPeluSxv28LmZPdxkA & Fiv5 & 380000 \\
\hline UC7TbCjfnW7bYDUoSRmEE99g & Guimar & 348000 \\
\hline UCmEv-tZEct7NE_IK29tbJEw & AtiveGamers & 310000 \\
\hline UCK_RIUd4z4itN7dt-ro9qVw & sharkjmp & 230000 \\
\hline UCiz-NHCExb8Q1zmvy0An4ug & Canal do Saullo & 226000 \\
\hline UCJVBLi-f4YsVppWI6tNKCcg & Isabela Barri & 182000 \\
\hline UC_GqAJpzUzTkXzTIXBYXk1w & Combo Infinito & 163000 \\
\hline UCAfL3j-ieND4ntVWVxMSWOg & AkrinuSS & 152000 \\
\hline UCGDI3T92XGq6SKH-1LSb9Fw & FIFA TRADE BRASIL & 134000 \\
\hline
\end{tabular}




\begin{tabular}{|c|c|c|}
\hline UCAeQOTxq6nDf4tLp9LSyH5Q & Canal do AEG & 122000 \\
\hline UCZv_YuZQ6MoDOlb3QR2IzjA & Games das antigas & 117000 \\
\hline UCVGjknmUTXDKptvmQoiHYjw & Noob Opressor & 108000 \\
\hline UCynu74RjF8sOncdnu0XadPw & CANAL DO WILL & 96400 \\
\hline UCs1H3tWffatW3zHRJbpFmYA & marvadogamer & 63300 \\
\hline UC_j3xI3yIMiskLHPv2yYQ0w & FullBuster & 33100 \\
\hline UCpwwdox2iSmv9VrHsR4evxA & Defenestrando Jogos & 31500 \\
\hline UCWwpCANjklMXPMLjeKohAnA & Noisquevoa Bruxao & 27300 \\
\hline UCXSwgwFSF5lv6avL1NxDVSQ & Erik Brasil - TodayPlays & 19800 \\
\hline UCLNTVBPHsNWgIQNuQyC4VDQ & João McFly & 11200 \\
\hline UCAlytYGGNJh5Xgkn6sxHM5A & Clã Ura & 7700 \\
\hline UCcmy-ukPFuH1Pb4G-1dWEGw & $\mathrm{rnSx}$ & 2590 \\
\hline UCo1xKQdc-DKD_u1m00YtWJw & Primeiro Player & 2180 \\
\hline UC5q5ptFREB7ERcvcjBpWbvw & AVG Mobile & 2020 \\
\hline
\end{tabular}




\begin{abstract}
The following article aims to analyse a possible politicization of vídeos about vídeo games based on the YouTube algorithm, through a methodological approach that combines the topographic analysis of the platform's recommendation networks and qualitative analysis of the videos that could be responsible for linking the theme with more radicalized videos in general. The proposal is to test the hypothesis of a radicalization led by the YouTube algorithm. Contrary to what we expected, the platform proved to be very capable of dividing its recommendations thematically, with little penetration of politicized vídeos in the network. However, strategies of more politicized (and radicalized) groups were used as recommendation paths.
\end{abstract}

Keywords: YouTube; radicalization; recommendation algorithms; extreme right.

\title{
Resumen
}

El siguiente trabajo busca analizar una posible politización de videos sobre videojuegos basados en el algoritmo de YouTube. Mediante un enfoque metodológico que combina el análisis topográfico de las redes de recomendación de la propia plataforma, y un análisis cualitativo de videos que podrían ser los encargados de vincular el tema con otros más radicales. La propuesta es probar la hipótesis de que existe un proceso de radicalización política liderado por el algoritmo de YouTube. Al contrario de lo que esperábamos, la plataforma demostró ser muy capaz de dividir temáticamente sus recomendaciones, con poca penetración de videos politizados en nuestras extracciones. Sin embargo, se utilizaron estrategias de grupos más politizados para ubicarse entre espacios de recomendación. politizados foram utilizadas para se colocarem entre espaços de recomendação.

Palabras clave: YouTube; radicalización; algoritmos de recomendación; extrema derecha. 